\title{
The ciliary pocket
}

\author{
Alexandre Benmerah ${ }^{1,2,3}$
}

\author{
Affiliations: \\ ${ }^{1}$ INSERM U1016, Institut Cochin, Paris, France. \\ ${ }^{2}$ CNRS UMR8104, Paris, France. \\ ${ }^{3}$ Université Paris Descartes, Sorbonne Paris Cité, Paris, France.
}

Address for correspondence: alexandre.benmerah@inserm.fr Institut Cochin, 22 rue Méchain, 75014, Paris, France.

Tel :33 140516578 ; fax : 33140516570 


\begin{abstract}
:
Cilia are fascinating highly conserved organelles shared by very different organisms from unicellular eukaryotes to vertebrates where they are involved in motility and sensory functions. In vertebrates, the function of the primary cilium, a unique non-motile cilium found at the surface of most cell types during development, remained mysterious during 40 years until its crucial function in the control of key signaling cascades during development and its involvement in complex genetic disorders now called ciliopathies were uncovered. Recent studies have focused on a specific membrane domain found at the base of primary cilia in most cell types which was already mentioned in the first descriptions of these cilia but did not raise much interest during 50 years. This membrane domain, the "ciliary pocket", also found at the base of some motile cilia, may act as a platform for cilia-associated vesicular trafficking and as interface with the actin cytoskeleton but also likely in additional important functions which remain to be discovered.
\end{abstract}


Cilia and flagella are highly conserved organelles which share a similar structural organization and biogenesis pathway $([1,2]$ and Fig.1). The axoneme, the microtubule-based skeleton of the cilium, is assembled from the basal body (BB). It is covered by the ciliary membrane which is in continuity with the plasma membrane (PM) but functionally separated from it by a diffusion barrier which limits the exchanges between the cilium and the rest of the cell. This diffusion barrier is the membrane-associated part of a more complex structure which is made by the transition fibers, the distal appendages of the BB, which interact with the PM, and the transition zone, linking the very proximal part of the axoneme with the ciliary membrane $[3,4]$. The import of ciliary components and their transport along the axoneme is mediated by the intraflagellar transport (IFT) machinery. IFT particles are believed to select cargoes to be transported from the cytoplasm and IFT-cargo complexes are transported by kinesin II from the BB toward the ciliary tip where they dissociate. IFT particles and kinesin II motor are transported back to the BB by cytoplasmic dynein $2[5]$.

In vertebrates, cilia have been classified into two functional families, motile and sensory cilia, however motile cilia can also be involved in sensory functions [6]. They are usually distinguished based on the organization of their axonemes, while both present 9 peripheral doublets of microtubules but motile cilia present an additional central pair of microtubules $(9+0$ versus $9+2$ cilia, respectively) as well as other specific structures required for their motility. Non-motile sensory cilia are generally unique and involved in signaling functions [7]. The first sensory cilium to be characterized at the ultrastructural level was the modified cilium forming the outer segment of photoreceptors [8]. Cilia with a similar organization have been soon after described in many if not all tissues examined and this structural similarity led to the hypothesis that they could be involved in sensory functions. They were called primary cilia (PC) by Sorokin because they are the first to appear during development [1]. The functions of these cilia remained mysterious until 2000 when it was established that their dysfunction causes polycystic kidney disease [9]. It is now clear that PC control key signaling pathways during development (hedgehog, PCP) and mutations of genes encoding for proteins required for their function and/or assembly cause complex genetic disorders now called ciliopathies [10].

\section{0 years after the primary cilium, the re-birth of the ciliary pocket}

Even if PC in different cell types show an identical skeletal organization, initial characterization of PC by transmission electron microscopy (TEM) revealed a striking difference in terms of membrane 
organization at their base. Indeed, PC in beta cells of the pancreas [11] and in secretory cells of the adenohypophysis ([12] and Fig. 1d) are deeply rooted in the cytoplasm with their BB pulling the PM which then forms a tubular structure surrounding the proximal part of the cilium. In kidney tubules epithelial cells, PCs directly protrude from the apical membrane with their BB docked within the same plane as the rest of the PM ([13] and Fig.1a-c). This differential membrane organization was also pointed by Sorokin who analyzed ciliogenesis in various tissues during development, and these differences drove him to propose two distinct pathways for primary ciliogenesis [1]. PC can form either by direct docking of the BB to the PM (epithelial cells, in green in Fig.1), or by a complex process during which the BB first docks onto a cytoplasmic vesicle inside which the axoneme grows, the cilium being partially released at the cell surface upon fusion of this vesicle with the PM (fibroblasts, in blue in Fig.1). At this latter step, the incomplete release of the cilium is likely to explain the presence of the tubular "invagination" of the PM at the base of PC observed in various cell types [14].

The presence of a membrane domain at the base of PC was progressively forgotten, even after the regain of interest for this fascinating organelle. One of the reasons for this neglecting is likely that kidney cells have been the cellular model at the base of this rebirth [9]. However, some studies suggested possible important and conserved functions for this membrane domain. Indeed, detailed analysis of PC in connective tissues suggested its possible role in cilium-associated vesicular trafficking [15]. In addition, a similar membrane domain was characterized at the base of the flagellum of trypanosomatids, this "flagellar pocket" (FP) is the unique site for endocytosis and exocytosis in this organism [16].

With these observations in mind, three publications in 2010 contributed to the regain of interest for this membrane domain. Altogether, they provided a detailed characterization and evidences for its presence at the base of all types of cilia in mammals (flagellum, motile and primary cilia). We called this membrane domain the "ciliary pocket" (CiPo), due to its morphological and functional similarity with the FP [17]. Rohatgi and Snell used the same name but to specifically describe the proximal part of the overall membrane domain [18]. Finally, Rattner and colleagues called this domain the "cilium pit" [19], but this term was not further used by others. The name CiPo was thereafter used in recent studies and reviews to describe the overall membrane domain.

These studies and pictures for past observations revealed that the CiPo is characterized by the presence of budding clathrin-coated pits (CCPs, Fig.2a) suggesting its function in endocytosis similarly as for the FP in trypanosomatids [17]. In addition, the CiPo is a docking site for actin cables (Fig.2b, [17,19]), an interesting observation to be paralleled with the recent findings which evidenced that actin 
polymerization negatively controls ciliogenesis [20-22]. Altogether, these observations suggested important and conserved functions for this membrane domain which has intrigued the cilium community.

\section{Morphogenesis}

At its proximal end, i.e close to the $\mathrm{BB}$, the CiPo starts at the docking site of transition fibers, the membrane of the CiPo is there almost perpendicular to the cilium axis but rapidly folds toward and along the cilium to surround its proximal part. There, the pocket can adopt different shapes depending of the cell type. In most cells, the membrane of the CiPo seems to establish contacts with the ciliary membrane and form a narrow tube with a diameter smaller than the more proximal "bulging" part, the CiPo then adopts a champagne cork like shape (Fig. 2d). An elegant recent study using tomography provided nice $3 \mathrm{D}$ view of such pockets in endothelial cells [23]. In other cell types, the CiPo membrane does not appear to establish contact with the ciliary membrane adopting then either a conic (IMCD3 cells, Fig. 2f) or tubular shape (cumulus cells, Fig. $2 \mathrm{~g}$ ). The length of the pocket, form its proximal end to the cilium exit site, can vary between cell types [17] but also within the same cell type as first noticed by B. Barnes [12].

The presence of the CiPo is likely to result from the fusion of the cilium containing vesicle with the PM $[1,14,17,18]$. Therefore, one could ask why the cilium is not completely released from its vesicle after fusion with the PM? First, physical links between BB/centrosome and the nucleus [24] might prevent its migration toward the plane of the PM. Second, interactions between the ciliary and CiPo membranes might also contribute to prevent the complete release of the cilium. Interestingly, perturbation of actin polymerization results in the modification of the shape of the CiPo which becomes wider with a loss of interaction between the two membranes ([19] and Fig.2, $d$ and e). This acute enlargement of the CiPo does not seem to result in the complete release of cilia suggesting that it is controlled by other mechanisms.

These latter data however indicate that actin polymerization controls the shape of the pocket. Actin polymerization regulates the activity of adhesion molecules [25] suggesting that adhesion proteins on both sides might mediate the interaction between CiPo and ciliary membranes. Therefore, modification of actin polymerization, lack of expression or differential targeting of one or both of the adhesion partners is likely to result in the loss of interaction between the two membranes and therefore in cell types specific pocket shapes. Finally, secretion of proteins inside the lumen of the CiPo can increase local 
turgor pressure and therefore again results in cell type specific and/or cell activity dependent modifications of the shape of the CiPo.

From TEM studies it is clear that the CiPo is in continuity with the PM $([17,19]$ and Fig.2d). In addition, there is no evidence for a structure similar to the "flagellar pocket collar", a cytoskeleton-based structure which restricts the exchanges between the PM and the FP in trypanosomatids [16]. In agreement with the absence of a diffusion barrier at the distal end of the pocket, the CiPo membrane seems to be a subdomain of the PM. First, it is enriched for CCPs with a similar composition as to PM-specific CCPs and those CCPs are able to capture transferrin as all the other PM-associated CCPs. Second, the CiPo membrane is stained by different GFP-fusions widely used as specific markers of the PM such as farnesylated-GFP [17] and PI(4,5)P2-specific GFP-fusions (unpublished observations).

Whether the CiPo membrane is a homogenous domain or if functional subdomains co-exist is still an open question. CCPs are mostly found along the tubular part [17]. The high curvature of the bulging domain might prevent assembly/budding of CCPs from this region of the CiPo. It might also favor a different lipid and protein composition of this domain [26]. This subdomain is therefore likely to correspond to the periciliary region [27] which was described as a membrane domain at the base of PC in kidney epithelial cells with specific lipid composition [28]. However, there is no direct evidence for a similar domain at the base of CiPo-associated cilia.

\section{Function(s) for the ciliary pocket?}

The fact that the CiPo is enriched in CCPs of course suggests its possible role in endocytosis but what can be the specific function for endocytosis here? Despite of the fact that CiPo-associated CCPs are functional and dynamics, clathrin-mediated endocytosis is not required for ciliogenesis nor for the general organization of the CiPo itself [17]. It remains possible that endocytosis at the CiPo is involved in internalization of cargo coming from the ciliary membrane or to the elimination of excess of incoming ciliary membrane proteins, two hypothesis which remain to be tested. Interestingly, an endocytic zone with a different organization is also present at the base of sensory cilia in C Elegans. There, clathrinmediated endocytosis appears to be involved in the targeting of ciliary and IFT proteins to the cilium [29]. It remains to be tested if cilia-associated endocytosis is involved in similar function at CiPoassociated PC in vertebrates. 
In addition to endocytosis, it is tempting to speculate that the CiPo could be a site for docking and fusion of secretory vesicles for the specific delivery of ciliary membrane proteins $[14,30]$. Interestingly, the CiPolike domain present at the base of the connecting cilium is indeed a site for docking of IFT-positive vesicles [29]. In addition, the early images obtained by Barnes and Munger clearly show the accumulation of secretory granules close to the CiPo $[11,12]$ suggesting its possible role in polarized secretion in specialized cells. Interestingly, recent studies using tomography or serial sectioning TEM revealed that the CiPo membrane is connected to tubular structures [23,31]. It would be interesting to determine if these structures correspond to alternative flues toward the PM or to conducts toward intracellular compartments for secretion within the CiPo lumen/membrane.

Comparing the cell types where cilia are associated or not with a CiPo, it is clear that PCs without pockets are present at the apical membrane of epithelial cells (kidney tubules [13], bile ducts [32]), where they are sensing flux of liquids [7], whereas CiPo-associated PC are mostly found in cells present within cell types where they are very unlikely to be involved in similar functions [14]. Interestingly, CiPo-associated PC are found in cells from connective tissues where they are supposed to sense mechanical stresses. The CiPo might therefore act there as a membrane reservoir like a spring, an amplifier or transducer of forces toward the cilium. In addition, in in vitro cultured non polarized cells, PC- associated CiPo are not randomly positioned and are parallel to the adherent surface $[17,33]$, an observation in agreement with the non-random positioning of PC in some tissues, including in connective tissues [34]. It is therefore tempting to speculate that the CiPo somehow participate in the positioning of PC, and likely through its connection with the actin cytoskeleton.

With all these observations and questions in mind, I was recently stricken by the morphological similarity of the CiPo with the hair follicle, the structure in which the hairs are rooted in the skin (Fig.3). Indeed, the growing region of the hairs is found within the dermis and is there surrounded by an invaginationlike of the epidermis named the root sheath. Interestingly, this pocket-like structure is connected to an actin-based structure, the arector pili muscle which control stand up of the hairs and is also the site for secretion of the sebum from the adjacent sebaceous glands [35]. The hairs are not randomly rooted in the dermis by a complex process controlled by the PCP pathway which control their coordinated orientation at the surface of the skin [36]. In addition, hairs are part of a complex network of structures involved in touch sensing in the skin [37] and their specific orientation plays a role in their sensory functions, indeed, everybody can feel that hairs transduce distinct sensations in response to stimuli in the same orientation or in the reverse direction. Therefore, one of the functions of the CiPo could be to 
ensure a specific orientation of the cilium for directional sensing. Whether the CiPo directly participates in the orientation of PC or only surrounds the cilium deeply rooted an oriented by other means is then an important question for future investigations. 


\section{Acknowledgments:}

First of all, I would like to apologize to the authors whose work was not discussed or cited owing to space limitations. I would like to thank Anahi Molla-Herman, Rania Ghossoub, Julia Hernandez-Rapp and the other former students in my lab who did most of the work on the ciliary pocket; Patricia Bassereau for stimulating discussions; Philippe Bastin and Thierry Blisnick for scanning EM picture in Fig.1; Lionel Larue and Delphine Champeval for the picture of mouse hair follicles in Fig.3. Work in the laboratory is supported by INSERM, CNRS, Université Paris Descartes and grant from Agence Nationale de la Recherche (ANR, R09087KS). 
Figure legends:

Figure 1: Primary ciliogenesis.

Left, scheme showing the organization of the primary cilium (PC) and of the intraflagellar transport (IFT). Right, description of the primary ciliogenesis pathways as first proposed by Sorokin in 1968 [1]. In green, the "extracellular pathway" which takes place in polarized epithelial cells where the basal body (BB) docks at the apical plasma membrane from where the cilium directly protrudes. $(a, b)$ TEM pictures from Latta and collaborators showing longitudinal section of PC in kidney tubules epithelial cells ([13], (C) 1961 Rockefeller University Press). (c) Scanning EM picture showing a PC at the apical surface of IMCD3 kidney cells. In blue, the "intracellular pathway" which takes place in fibroblasts where the basal body interacts with a "primary ciliary vesicle", the axoneme grows within this vesicle which elongates and then forms the ciliary sheath (blue) surrounding the ciliary shaft (i.e. the ciliary membrane, red). The cilium containing vesicle finally fuses with the plasma membrane allowing the cilium to reach the cell surface. This fusion event results in the formation of a membrane domain which surrounds the proximal part of the cilium, the ciliary pocket. (d) TEM picture from B.G. Barnes showing a longitudinal section of a primary cilium in secretory cells of the mouse adenohypohysis (reprinted from [12] with permission from Elsevier).

\section{Figure 2: The ciliary pocket.}

Top panels, TEM pictures of longitudinal sections of PC in RPE1 cells (retina pigmented epithelial cells) showing clathrin-coated pits (CCPs, (a)) budding from the ciliary pocket membrane (blue arrows) and actin filaments (b) lying close to the ciliary pocket. (c) Scheme showing the general organization of the ciliary pocket (blue), an endocytic membrane domain from where clathrin-coated pits (CCPs) and vesicles are formed and also involved in interaction with the actin-based cytoskeleton. The ciliary pocket may also act as a platform for docking and fusion of secretory vesicles for the delivery of cargos en route for the ciliary membrane. Bottom panels, TEM analysis of the ciliary pocket in RPE1 cells not treated (d) or treated $(e)$ with cytochalasin-D to perturb the polymerization of actin. TEM pictures of longitudinal 
sections of PC in in vitro cultured IMCD3 kidney cells (f) and of cumulus cells from oocytes collected from hyper ovulating mice $(g)$.

\section{Figure 3: the root sheath of hair follicles, a multicellular pocket-like structure.}

The general organization of ciliary-pocket associated cilia is similar to that of the hair follicle in which hairs are rooted. (a) TEM pictures showing a complete longitudinal section of a primary cilium in RPE1 cells. (b) Hematoxylin and eosin staining of mouse skin showing a complete longitudinal section of a hair follicle. (c) Scheme showing the organization of the hair follicle. The hair is rooted within a depression of the epidermis, the root sheath (inner and outer), which can be compared to the ciliary pocket (Fig.2c). Sebaceous glands are connected to the root sheath in which they secrete their content (sebum). In addition, the root sheath is the site of docking of the arector pili muscle (actin based structure) and also exert sensory functions through specific innervation. 


\section{References}

**1. Sorokin SP. Reconstructions of centriole formation and ciliogenesis in mammalian lungs. J Cell Sci $1968 ; 3: 207-230$.

Detailed analysis of ciliogenesis in mammals, models for ciliogenesis and the name "primary" cilium is given to $9+0$ and two centrioles basal bodies cilia.

2. Ishikawa H, Marshall WF. Ciliogenesis: building the cell's antenna. Nat Rev Mol Cell Biol 2011;12:222-234.

3. Hu Q, Nelson WJ. Ciliary diffusion barrier: the gatekeeper for the primary cilium compartment. Cytoskeleton (Hoboken) 2011;68:313-324.

4. Reiter JF, Blacque OE, Leroux MR. The base of the cilium: roles for transition fibres and the transition zone in ciliary formation, maintenance and compartmentalization. EMBO Rep 2012;13:608618.

5. Pedersen LB, Rosenbaum JL. Intraflagellar transport (IFT) role in ciliary assembly, resorption and signalling. Curr Top Dev Biol 2008;85:23-61.

6. Bloodgood RA. Sensory reception is an attribute of both primary cilia and motile cilia. J Cell Sci 2010;123:505-509.

7. Satir P, Christensen ST. Overview of structure and function of mammalian cilia. Annu Rev Physiol 2007;69:377-400.

*8. DE ROBERTIS E. Morphogenesis of the retinal rods; an electron microscope study. J Biophys Biochem Cytol 1956;2:209-218.

**9. Pazour GJ, Dickert BL, Vucica Y, Seeley ES, Rosenbaum JL, Witman GB, Cole DG. Chlamydomonas IFT88 and its mouse homologue, polycystic kidney disease gene tg737, are required for assembly of cilia and flagella. J Cell Biol 2000;151:709-718.

First evidence that polykystic kidney disease is linked to primary cilium dysfunction.

10. Hildebrandt F, Benzing T, Katsanis N. Ciliopathies. N Engl J Med 2011;364:1533-1543. 
11. MUNGER BL. A light and electron microscopic study of cellular differentiation in the pancreatic islets of the mouse. Am J Anat 1958;103:275-311.

**12. BARNES BG. Ciliated secretory cells in the pars distalis of the mouse hypophysis. J Ultrastruct Res 1961;5:453-467.

First detailed description of cilia with an organization similar to the connecting cilium of photoreceptors and hypothesis that these cilia might be involved in sensory functions.

13. LATTA H, MAUNSBACH AB, MADDEN SC. Cilia in different segments of the rat nephron. J Biophys Biochem Cytol 1961;11:248-252.

*14. Ghossoub R, Molla-Herman A, Bastin P, Benmerah A. The ciliary pocket: a once-forgotten membrane domain at the base of cilia. Biol Cell 2011;103:131-144.

Review focusing on the ciliary pocket, its biogenesis, distribution and possible functions.

*15. Poole CA, Flint MH, Beaumont BW. Analysis of the morphology and function of primary cilia in connective tissues: a cellular cybernetic probe? Cell Motil 1985;5:175-193.

Analysis of PC in connective tissues and discussion about PC-associated vesicular trafficking.

16. Field MC, Carrington M. The trypanosome flagellar pocket. Nat Rev Microbiol 2009;7:775-786.

**17. Molla-Herman A, Ghossoub R, Blisnick T, Meunier A, Serres C, Silbermann F, Emmerson C, Romeo K, Bourdoncle P, Schmitt A, Saunier S, Spassky N, Bastin P, Benmerah A. The ciliary pocket: an endocytic membrane domain at the base of primary and motile cilia. J Cell Sci 2010;123:1785-1795.

Fisrt morphological and functional description of the ciliary pocket at both primary and motile cilia.

**18. Rohatgi R, Snell WJ. The ciliary membrane. Curr Opin Cell Biol 2010;22:541-546.

Review article on the organization of membranes at cilia. Description of the ciliary pocket and proposed model for its biogenesis and function.

19. Rattner JB, Sciore P, Ou Y, van der Hoorn FA, Lo IKY. Primary cilia in fibroblast-like type B synoviocytes lie within a cilium pit: a site of endocytosis. Histol Histopathol 2010;25:865-875.

Description of the "cilium pit" in synoviocytes. 
20. Kim J, Lee JE, Heynen-Genel S, Suyama E, Ono K, Lee K, Ideker T, Aza-Blanc P, Gleeson JG. Functional genomic screen for modulators of ciliogenesis and cilium length. Nature 2010;464:1048-1051.

21. Pitaval A, Tseng $Q$, Bornens $M$, Théry M. Cell shape and contractility regulate ciliogenesis in cell cycle-arrested cells. J Cell Biol 2010;191:303-312.

22. Bershteyn M, Atwood SX, Woo W-M, Li M, Oro AE. MIM and cortactin antagonism regulates ciliogenesis and hedgehog signaling. Dev Cell 2010;19:270-283.

*23. Geerts WJC, Vocking K, Schoonen N, Haarbosch L, van Donselaar EG, Regan-Klapisz E, Post JA. Cobblestone HUVECs: a human model system for studying primary ciliogenesis. J Struct Biol 2011;176:350-359.

Description of ciliogenesis using 3D tomography in endothelial cells.

24. Razafsky D, Hodzic D. Bringing KASH under the SUN: the many faces of nucleo-cytoskeletal connections. J Cell Biol 2009;186:461-472.

25. Maruthamuthu V, Aratyn-Schaus Y, Gardel ML. Conserved F-actin dynamics and force transmission at cell adhesions. Curr Opin Cell Biol 2010;22:583-588.

26. Callan-Jones A, Sorre B, Bassereau P. Curvature-driven lipid sorting in biomembranes. Cold Spring Harb Perspect Biol [Internet] 2011 [cité 2012 juill 13];3. Available from:

http://www.ncbi.nlm.nih.gov/pubmed/21421916

27. Nachury MV, Seeley ES, Jin H. Trafficking to the ciliary membrane: how to get across the periciliary diffusion barrier? Annu Rev Cell Dev Biol 2010;26:59-87.

*28. Vieira OV, Gaus K, Verkade P, Fullekrug J, Vaz WLC, Simons K. FAPP2, cilium formation, and compartmentalization of the apical membrane in polarized Madin-Darby canine kidney (MDCK) cells. Proc Natl Acad Sci USA 2006;103:18556-18561.

Description of a membrane domain with a specific lipid composition at the base of PC.

**29. Kaplan OI, Doroquez DB, Cevik S, Bowie RV, Clarke L, Sanders AAWM, Kida K, Rappoport JZ, Sengupta $\mathrm{P}$, Blacque $\mathrm{OE}$. Endocytosis genes facilitate protein and membrane transport in C. elegans sensory cilia. Curr Biol 2012;22:451-460.

Characterization of an endocytic domain at the base of sensory cilia in C. Elegans. 
30. Bloodgood RA. The future of ciliary and flagellar membrane research. Mol Biol Cell 2012;23:24072411.

31. Schrøder JM, Larsen J, Komarova Y, Akhmanova A, Thorsteinsson RI, Grigoriev I, Manguso R, Christensen ST, Pedersen SF, Geimer S, Pedersen LB. EB1 and EB3 promote cilia biogenesis by several centrosome-related mechanisms. J Cell Sci 2011;124:2539-2551.

32. Masyuk TV, Huang BQ, Ward CJ, Masyuk Al, Yuan D, Splinter PL, Punyashthiti R, Ritman EL, Torres VE, Harris PC, LaRusso NF. Defects in cholangiocyte fibrocystin expression and ciliary structure in the PCK rat. Gastroenterology 2003;125:1303-1310.

*33. Albrecht-Buehler G. Phagokinetic tracks of 3T3 cells: parallels between the orientation of track segments and of cellular structures which contain actin or tubulin. Cell 1977;12:333-339.

First evidence that PC are not randomly positioned during migration and relative to the adherent surface.

34. Farnum CE, Wilsman NJ. Axonemal positioning and orientation in three-dimensional space for primary cilia: what is known, what is assumed, and what needs clarification. Dev Dyn 2011;240:24052431.

35. Schneider MR, Schmidt-Ullrich R, Paus R. The hair follicle as a dynamic miniorgan. Curr Biol 2009;19:R132-142.

36. Devenport D, Fuchs E. Planar polarization in embryonic epidermis orchestrates global asymmetric morphogenesis of hair follicles. Nat Cell Biol 2008;10:1257-1268.

37. Delmas $\mathrm{P}, \mathrm{Hao} J$, Rodat-Despoix L. Molecular mechanisms of mechanotransduction in mammalian sensory neurons. Nat Rev Neurosci 2011;12:139-153. 


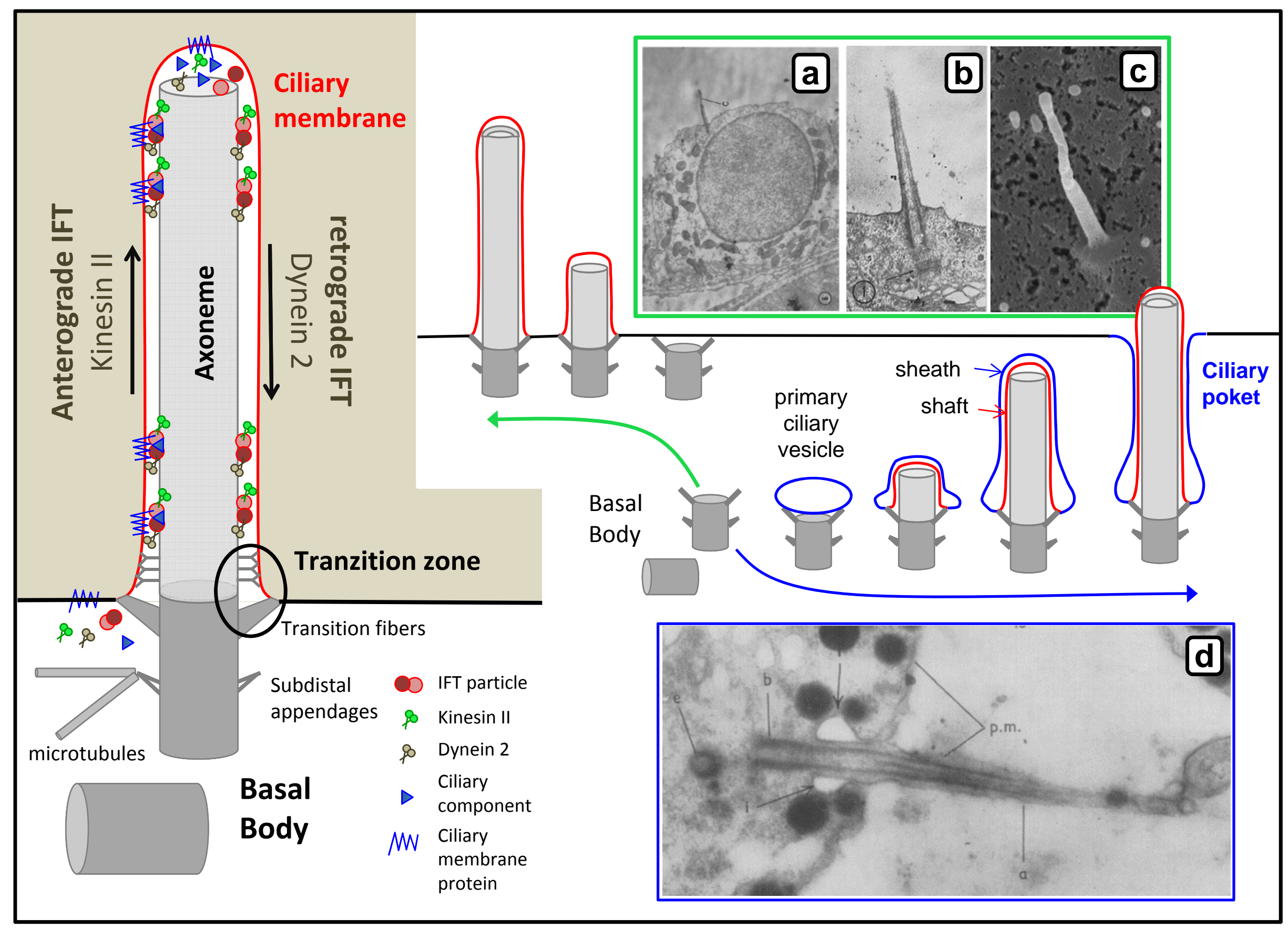



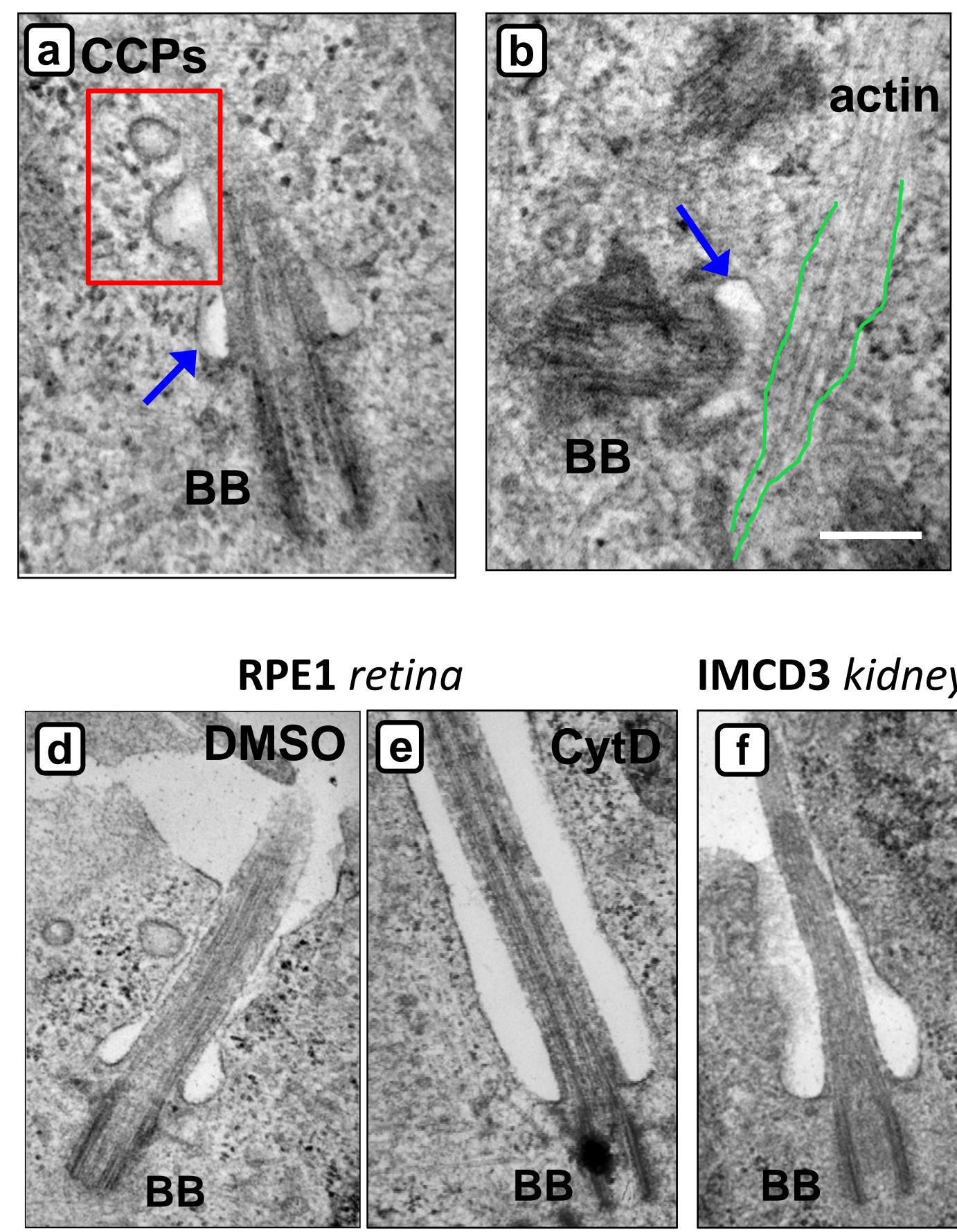

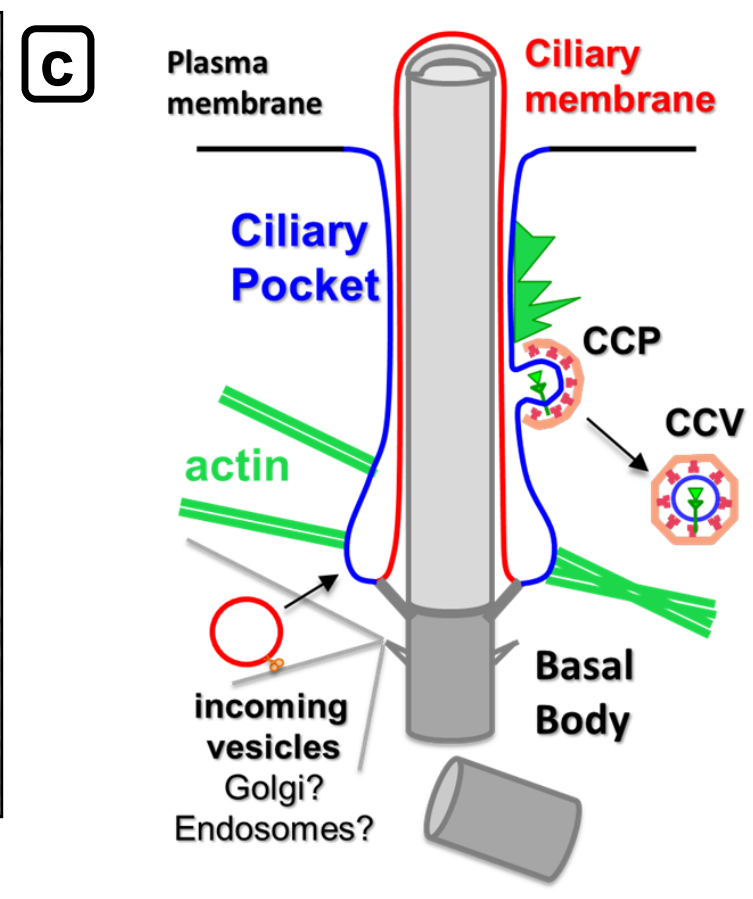

IMCD3 kidney Cumulus cells
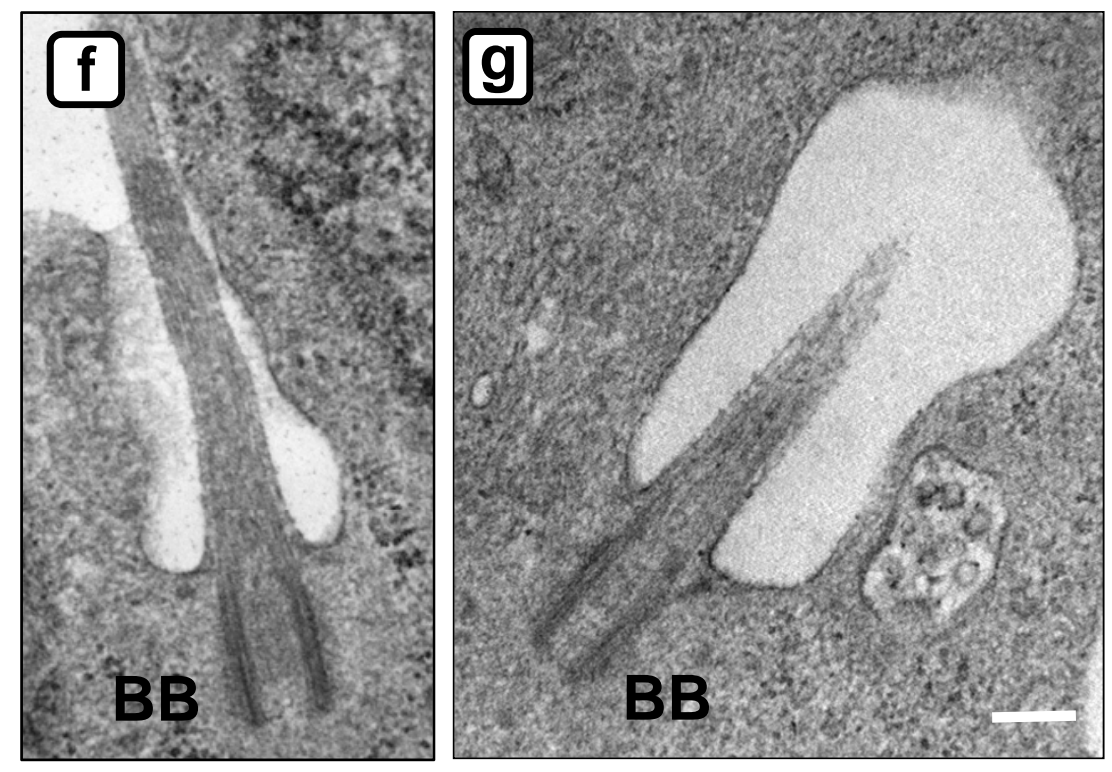


\section{Primary cilium (RPE1) Hair follicle (mouse)}

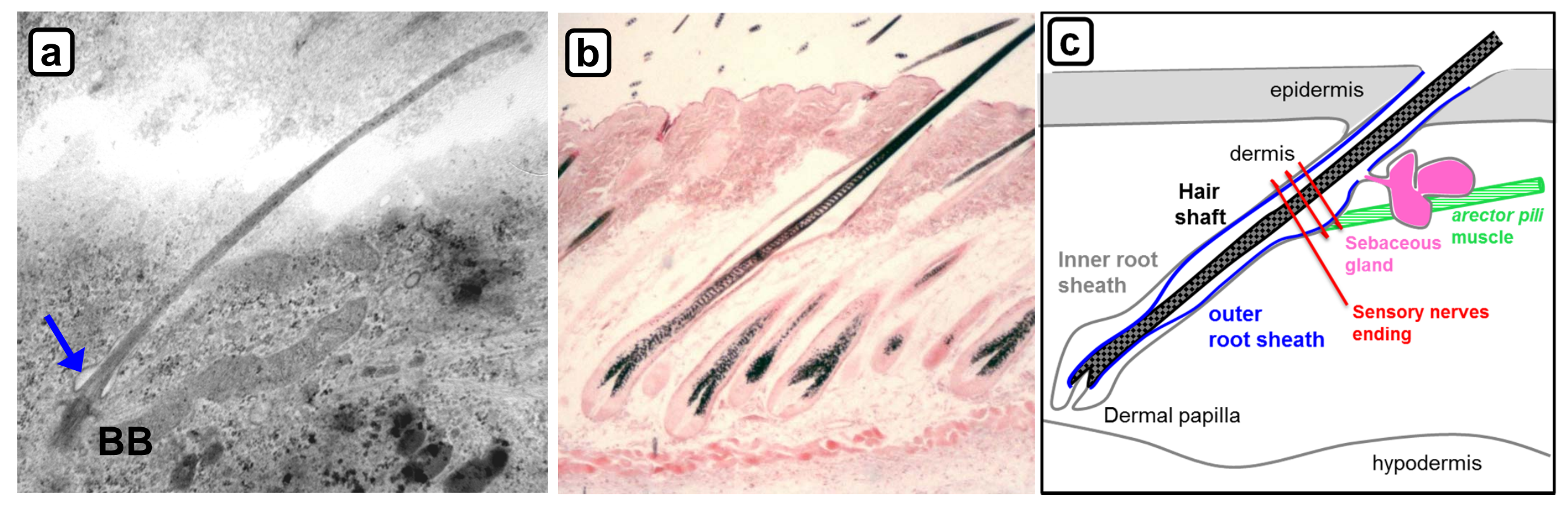

